\title{
The preliminary use of green building design in new residential Zhuang
}

\section{kuo flok in Qinghai ${ }^{\star}$}

\author{
Wang Jiameng ${ }^{1} \quad$ Jin Yibing ${ }^{2}$ \\ Fund Project: the National Natural Science Foundation of China (51208419) \\ About the Author: Wang Jiameng, Xi'an University of Architecture and Technology, Graduate, \\ 282360523@qq.com, China \\ Jin Yibing ${ }^{2}$, Xi'an University of Architecture and Technology, Associate Professor, \\ jinice1128@126.com, China
}

Keywords: Ecological security, zhuang kuo flok, Green Building, Update Design

Absract:Considering for the ecological security strategy and new promoting urbanization, it is important to develop the vernacular architecture in Tibetan plateau which serving as the ecological security barrier in China. With the development of urban and rural integration process as a whole and the popularit of the modernization conception,significant change happens in daily life style and also in production mode. This paper analyzes the yard layout,flat-type system and building materials of Zhuang kuo flok due to their widespred usage and unigue characterisics.Combing with green architecture elements,efforts were made to design new Zhuang kuo flok style building based on the regional features.

\section{Introduction}

Vernacular architecture is a kind of distinctive regional architecture with strong local characteristics of the traditional dwellings that is produced in a certain environment, which going through historical changes, the time flies, and the evolution of culture. Zhuang kuo flok is the representation of eastern Qinghai-Tibet plateau's folk house in Hehuang area, and its birth met with the basic needs for human. It was the results combining the region's geography resources, natural climate and cultural history, and it also represented a kind of traditional ecological residences that have adapt to cold weather conditions, the use of composite local technology, in unity of multi-ethnic region

However, under the progress of new urbanization, people's requirements of residential space and buildings are changing with the improvement of culture, economy and lifestyle level. And the extreme minds of developing have formed the conception of one-sided pursuit of money .These factors are neither good for living environment protection or the for development of residential buildings. Therefore, traditional houses like Zhuang kuo flok, which contain local characteristics and national characteristics, should be effectively protected and updated.These urgent problems need to be solved under the strategic context of ecology.

\section{The analysis of the ecological background in Hehuang area of eastern Qinghai}

The geographical overview

The Hehuang area,located in the northeast of Qinghai-Tibet plateau in Qinghai province, is a transition region of the Qinghai-Tibet plateau, the Inner Mongolia plateau and the Loess plateau. 
This region has a mixed geological condition with an average altitude of 2500 meters.

\section{The natural Features}

Hehuang area is cold, dry and windy with an annual average temperature at $6.9^{\circ} \mathrm{Crainfall}$ at about $350 \mathrm{~mm}$. There is strong solar radiation, long sunshine duration and large temperature differences. Windy spring, cool summer and dry winter in this area provid suitable condition for both agricultural and animal husbandry.

\section{The humanistic surroundings}

Hehuang area is one the earliest place where people live and work in the Yellow River area. With the development of trade and war, the different natural environments breed culture of farming and nomadic, and the Confucian culture from central plains, the Buddhism culture from northwest and Islam culture in couision and blend here. Therefore, the traditional Zhuang kuo flok with characteristic of cultural fusion came out in this area. (FIG.1)

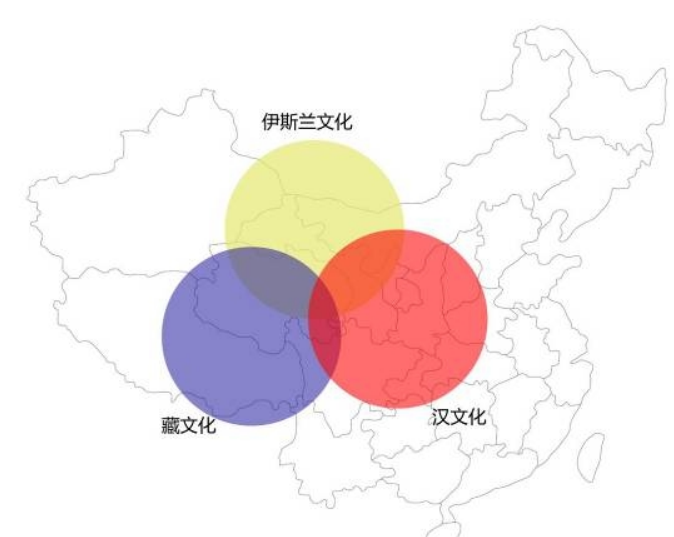

(FIG.1) Northwest multicultural enrichment zone (Owner-drawing)

\section{Zhuang kuo flok's features}

From the view of historical evolution and cultural exchange, the zhuang kuo flok has a natural integration with the regional characteristics. It is the product of Han Chinese courtyard architecture and Qiang Diao Fang blend of architecture.1 In Hehuang area, Zhuang kuo flok is selected by local minority residents in order to cope with climate change and technical suitability .Its courtyard is enclosed and regular, and the internal space is abundant. In response to the cold climate and technology mastery, Zhuang kuo flok use the local resources for constructions, which is economical, convenient and simple.

\section{Zhuang kuo flok's features}

The layout of the Zhuang Kuo Flok is enclosed shape structured, forceful thick, interior tidy beautiful, dignified and elegant. Zhuang Kuo Flok is characterized by having green energy, conservation of land, windbreak and cold-proof etc.. Zhuang Kuo Flok is combination of the main building, the atrium wall and courtyard. The formation of complete air spaces is insulation from circulation. Its tall walls could act as the defence for invasion and cold wind,as well as the resistance cold weather dgainst. Its enclosed courtyard space make the internal of Zhuang Kuo Flok has cool features. 


\section{The flat shape}

(1)"一" type plane: the main building plane is like "—” which is a Chinese word meaning 'one', and there have many rooms. Each room also ensures the best orientation and abundant sunshine., but generally the homestead area is larger, exposed the smallest, but its forming appearance are normal in architectural. (FIG.2)

(2)"L" key head type plane: "L" type plan can effectively block the sandstorm from northwest, and it can also effectively divide the architectural interior space. The subsidiary space is used to be temperature-damping region on the north side. Homestead area is small, in a land-saving made.

(3)" 凹 " type plane: This form not only effectively block the sandstorm from northwest, but also enlarge the sunshine corridor area and increase the activity of area in the corridor. The subsidiary space is used to be temperature-damping region on the north side. The form become more abundant and beautiful.(FIG.2)
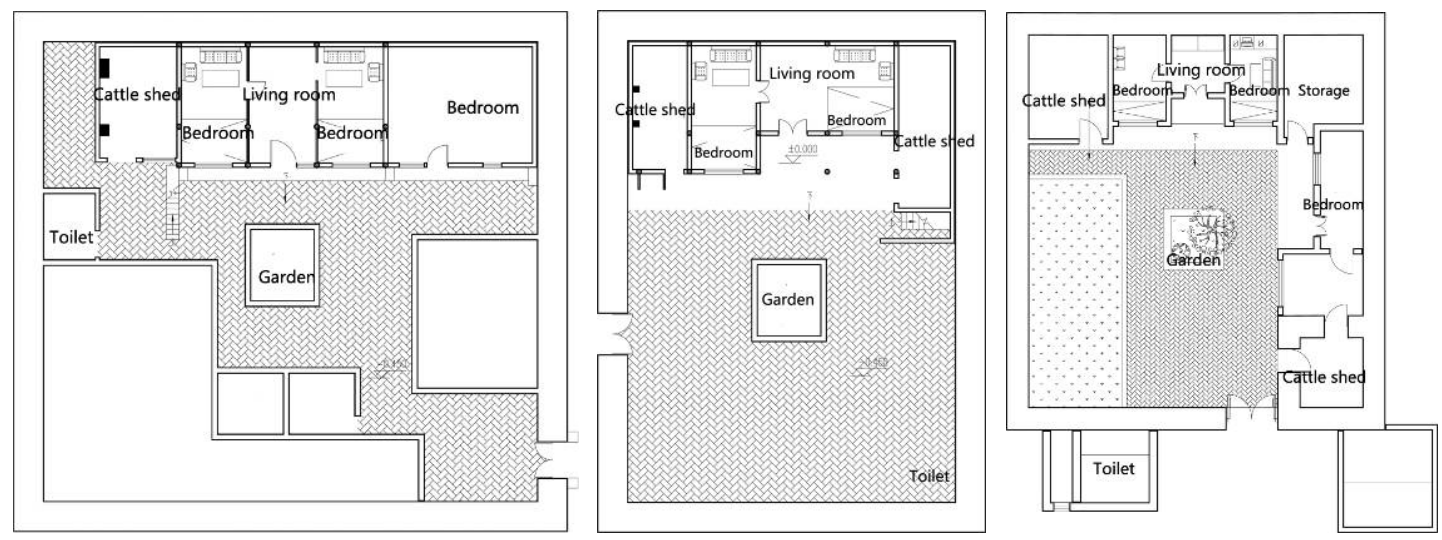

(FIG.2)
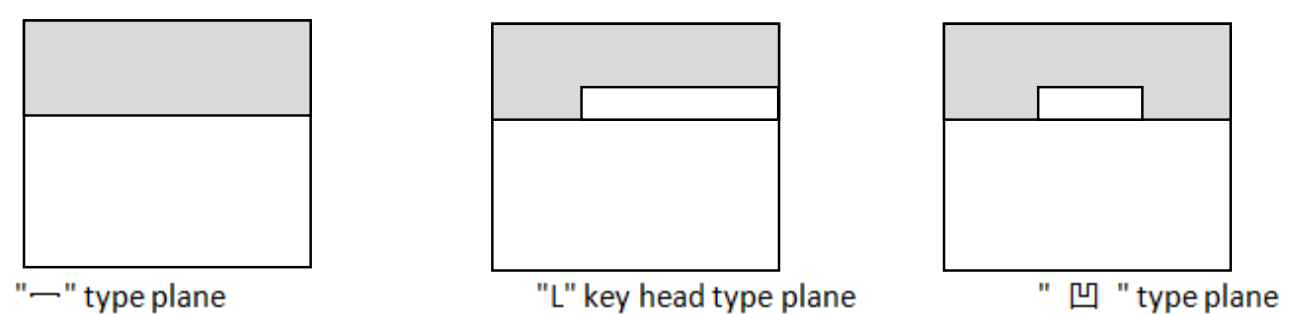

These three forms of residential areas that are mentioned above have been built in many areas in Hehuang region. With long history of change and adaptation to the environment, elements of mutual integration of all ethnic groups are reflected in the layout, showing one of the national cultures of pluralism.

\section{The building materials}

There are rich loess and oil resources in Qinghai Huangyuan areas, so most of the residents here choose these two materials as main materials for Zhuang Kuo folk single buildings. The walls were built with adobe and the gravel serbed as a base to prevent rain and tidal erosion. With such local material,it was possible to build green buildings which can meet geomorphological features, being biodegradable and energy-saving.

The loess is one kind of building materials that conform to the local climate conditions. (1) Thermal insulation: The local climate of morning and evening temperature difference is big, cold.As the maintenance ntenance structure rammed earth has its thermal insulation property. It has thermal insulation property. (2) Vigorous appearance: The construction blend with the earth in color, not making people feel the building on colour is not a kind of aggressive artificial structures. And the $1.6 \mathrm{~m}$ the Zhuang profile in thickness of the outer wall has a solid defensive performance. (3) 'Bone wooden' walls: Wooden bone mud wall of rammed earth buildings will collapse but not 
houses collapsed when the earthquake. (4) Biodegradable: As a kind of ,getting the from the natural materials from natural normalization to reduce construction waste generation, in could reduce the generation of construction waste.

Auxiliary material is stone in the local construction process. As auxiliary material, stone can concerning the fact that dilapidate village base and foundation are sometimes damaged more or less as a result of the rammed earth's intolerance against rain and wind.

\section{Problems faced by traditional Zhuang kuo flok}

According to the group's results of long-term observation analysis and field research, with the rapid development of urban and rural integration process, the increase rate of changes of people's lifestyle and advancing living standard has far exceeded the adaptability update rate of village gallery in natural form. There are some problems of traditional village gallery that need to be faced by the following aspects:

The functional differences

The traditional layout of the Zhuang kuo folk cannot meet the needs of modern life. Modern people require diverse functions such as a garage, entertainment room, guest room and so on.Thus, the traditional proportions and layout features need to be updated.

The low technical

'Zhuang Kuo flok' is eroded easily in rainy season, the corner of the outside wall is invaded by strong wind erosion perennially. The traditional technique of rammed earth construction of the wall and rammed earth material needs to be raised and processed to extend the life of rammed earth walls and increase its strength.

In the living habits of local residents, the lack of clean energy and secondary energy applications require the improvement of technology in order to make full use of energies such as solar and wind energy resources.

The lack of hygiene

The yak dung is a kind of convenient and economic fuel, but it is limited by season and output, And it is the source of illness of local people .Yak dung could be use to manufactured utilizing methane to provide clean energy.

Limited by the local conditions, residents here have formed the habit of perennial outdoor toilet using.Either from of health or life aspect, all that needs to be improved. For modern lifestyle, we need to gradually increase the sewage system and the construction of sanitary building within the traditional villages.

\section{The new residential green design strategies.}

Under the principle of the maximize land saving and the efficient use of natural resources, we should take advantages of the existing land space and saving resources. Under the circumstance of plain type and platform type,we try to create multi-parallel or single-family units, together with, applying a variety of the innovations into the design.(FIG.3、4) 


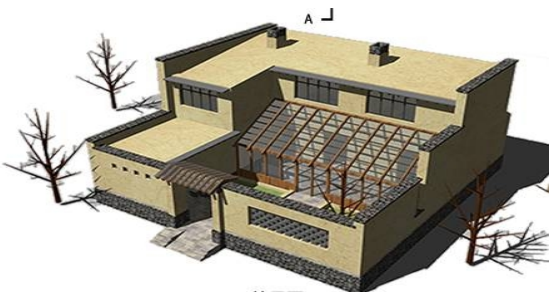

(FIG.3)one of new Zhuang kuo flok

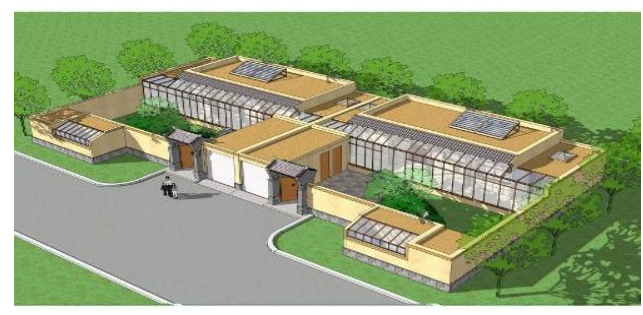

(FIG.4)one of new Zhuang kuo flok

\section{The optimized floorplan}

The new layout of Zhuang kuo folk are fine-tuning, based on the traditional Zhuang kuo flok.(FIG.5)

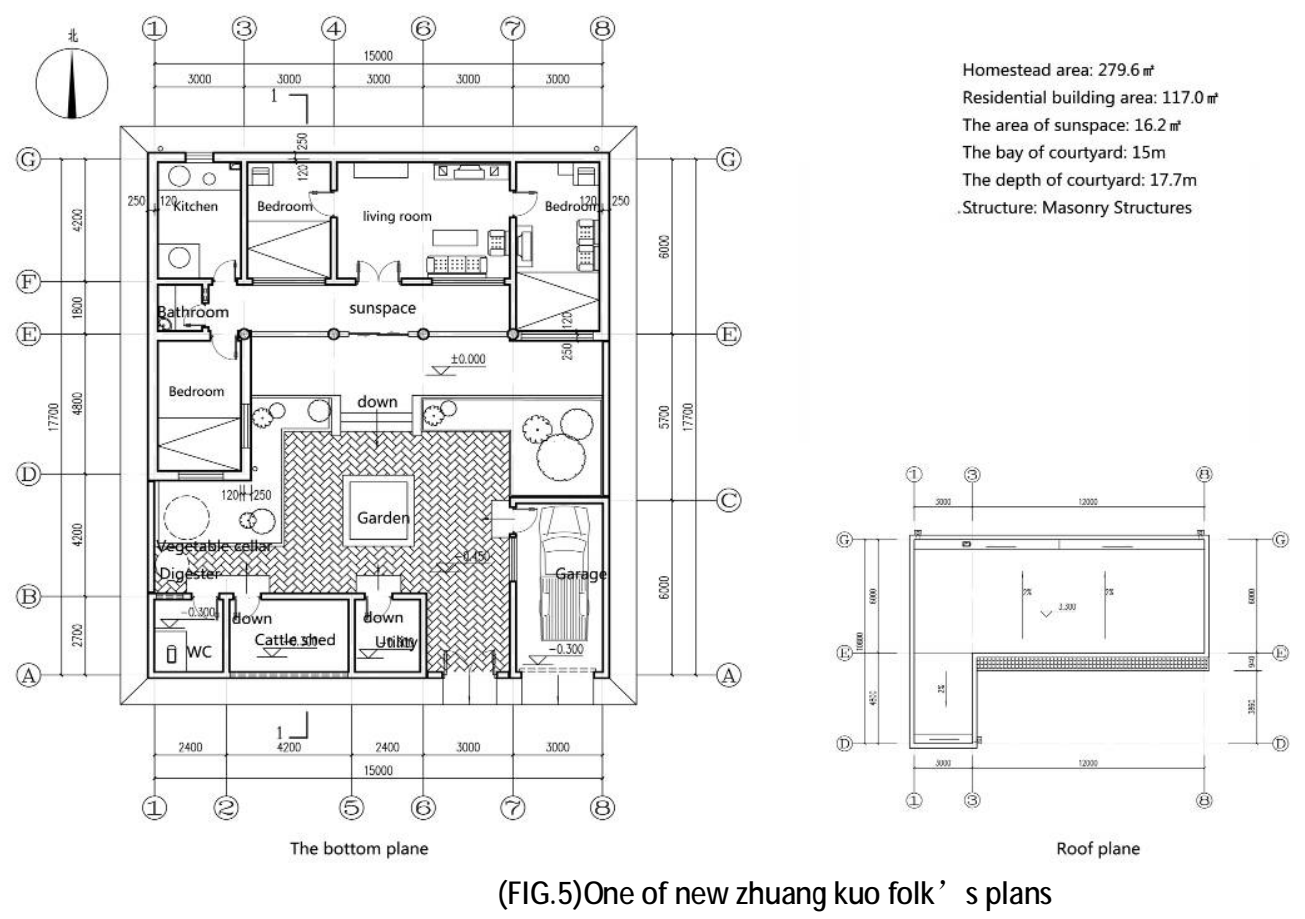

The main building and the west one are shapoed using the L-shaped pattern,which was placed in the atrium on the north side and the west side. The livestock sheds and garages logistics area are in L-shape, located in the south and the east side of the atrium. The main house is added with the bathroom functions, in order to solve the health problems of indoor toilets and showers, while In order to facilitate the production of biogas, outdoor toilets still be retained, but its location in combined with the legistical area,separating from the main room to solve, to solve health problems. And a garage is added at one side of the front door to satisfy the family's vehicle needs.

\section{The optimization of local materials}

Maintaining the principle without destroying the original style and green energy, and updateing materials with used rammed earth, straw bricks of other composite materials, instead of wood, can reduce the wall thickness, can also reduce wood consumption, without affecting thermal insulation properties.

\section{Optimization of construction techniques}

Rammed-earth wall construction technology was upgraded to an ecological technology of com posite wall combining traditional raw earth and concrete together.

Because the unsatisfied stability of raw earth and limited technical capacity, rammed-earth wall can not guarantee solidary for a longer term; as a result, coagulants, or 
other materials are added to the adobe, either the new wall hardness and strength has been enhanced, and producing a variety of wall texture effects, while improving the profile of the traditional village houses thick walls, which are easily ero ded by rain.So is it with the poor seismic performance and the monotonous building facade s.

\section{Usage of solar energy}

Rarely used solar cookers will be eliminated to reduce the waste of resources. After regenerating the roof with a reasonable slope, solar photovoltaic panels and solar water heater will be situatedon the roof. Additionally, a solar warm gallery will be constructed to have an efficient thermal conversion via solar energy.Set the sun gallery with the updating double vacuum low-e glass instead of the traditional single wooden-structured glass. And use certain methods to ensure the sun gallery's heat insulation and preservation.(FIG.6)

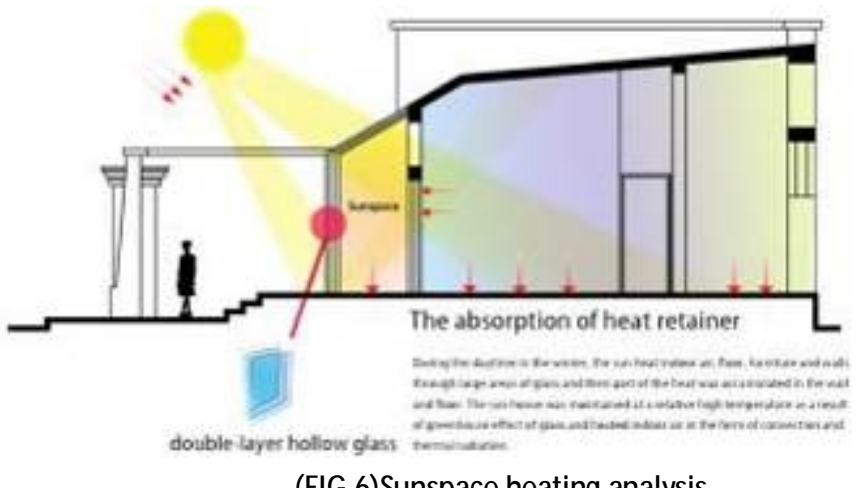

\section{External wall insulation and heating}

In graphic design, a layer of air in north side is carried out, which serves as the grid insulation layer damping. Two-story staircase is set in the north part, acting as air space to keep temperature steady.

Flue is longitudinally arranged in the house, and was caught in the middle of the two bedrooms on the second floor, is linked to the indoor heat kang. Smoke circulates into the floor cavity and roof cavities that are horizontally arranged on the second floor, and the finally discharged outside the building.Thus, the energy produced by the cow dung would be made full use of,creating a whole building finewall effects. (FIG.7)
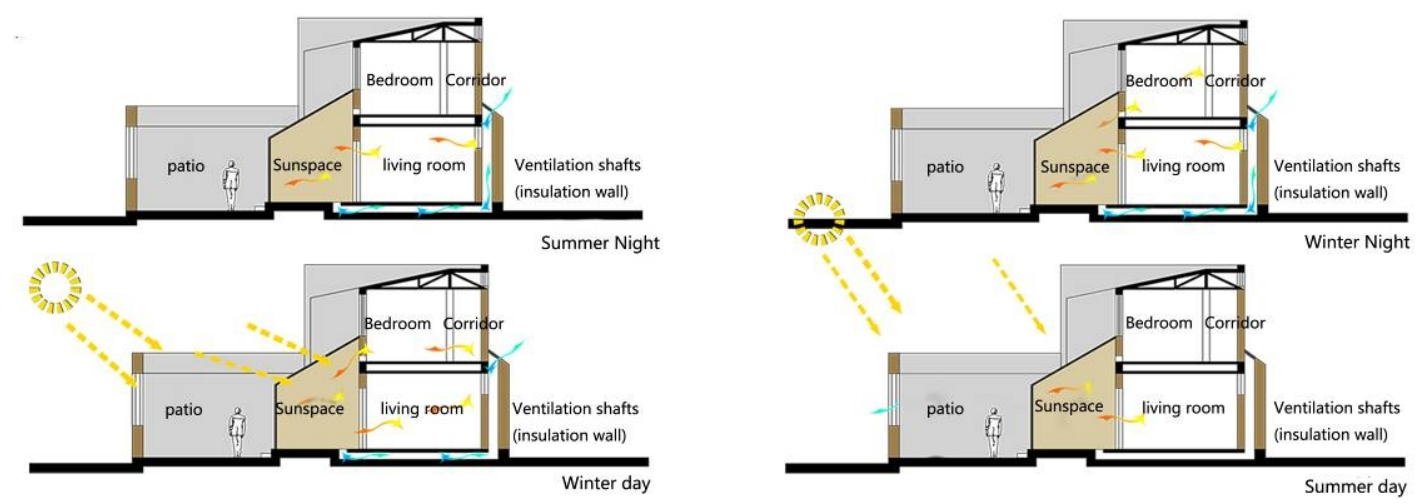

(FIG.7)The analysis isvenilation shafts and Sunshine Gallery for ventilation and lighting effects 


\section{Conclusion}

A new green architecture technology is adopted to update and activate the traditional Zhuang kuo flok buildings. Based on scientific investigation and analysis for traditional buildings, the new design showed another new way for traditional culture protection.

The research and update practice of zhuang kuo flok in Hehuang area help us understand much well of how to protect the traditional houses and how to upgrade the traditional Zhuang kuo flok to meet the needs of residents in modern society as quick as possible. The adoption of green building energy technique provides traditional Zhuang kuo flok new characteristics: high durability, high clean energy usage efficiency and other factors meeting almost all the needs for modern life.

\section{Reference:}

[1] Wang Jin Cui also river ice, etc. - Analysis and Regeneration of Traditional Houses Hehuang area $[\mathrm{J}]$ "New Construction" 2013/02.

[2] Wu Jingjing Wang Bai Tao - Eastern River in Qinghai Province and the traditional settlement area resident minority Huang Survival Study [J] volume "Sichuan Building Science" 2013/02 thirty-ninth the first period.

[3] Wang Cui river in Jan - Investigation and analysis of the traditional type of residential architecture resources under climate-oriented [J] "South Building" 2013/03

[4] Shaanxi Provincial Institute of Architectural Design Branch Qinghai - Huangyuan County, Qinghai Tibetan Village Balkans Rabbit township new rural planning and design specifications

[5] firewood bin - Qinghai complex ecosystem based Cacalia dry Villages Conservation and Development Strategy of Xi'an University of Architecture \& Technology 2015 works of art and design jobs

[6] Liu Xianjue - New Thinking protection and utilization of ancient dwellings

[7] Caishi Qian Chen Zhihui blue Xiao Ming - the use of traditional regional architecture of activation [J] "Huizhou University" 2010/12

[8] Dan Haiqing - Huangyuan County Cultural Industry Development of "climbing" 2014/04

[9] Wang Cui river - Generation and evolution of the traditional village in eastern Qinghai profile residential [J] Plateau forum.

[10] Wang $\mathrm{Yu}$ - Mountain tourism and cultural connotation and development [J] "Qinghai University for Nationalities" 2001/04

[11] http://baike.haosou.com/doc/5380903-5617192.html\#5380903-5617192-3 